\title{
Comparison of rotated paddle wheel reformation with standard axial CT scan in pulmonary embolism; a hospital based study
}

\author{
Hanaa Ahmed Kamel ${ }^{1}$, Hoda Salah Darwish ${ }^{2 *}$ and Sadia Raheez Qamar ${ }^{3}$ \\ *Correspondence: darwish.hoda@yahoo.com

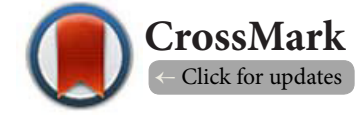 \\ ${ }^{1}$ Consultant Radiologist, Lecturer of Radio-diagnosis, Tanta University, Egypt. \\ ${ }^{2}$ Lecturer of Radio-diagnosis, Faculty of Medicine, Radiology Department, Suez Canal University, Egypt. \\ ${ }^{3}$ Specialist Radiologist, Department of Radiology, Rawalpindi Medical college, Pakistan.
}

\begin{abstract}
Paddle wheel technique is a reconstruction method of displaying images, which uses planar slabs pivot on the central horizontal axis at bifurcation of pulmonary artery trunk. With this method, each image depicts complete track of branching structures from central hilum to the periphery.

Objective: The purpose of this study was to assess the role of paddle wheel reformation and compare it with standard axial multidetector CT images in cases of pulmonary embolism.

Methods: CT scans of 50 patients presented for CT pulmonary angiography were selected and interpreted by two radiologists using reformatted paddle wheel technique besides standard axial CT scans. The scans were reviewed independently and randomly to reduce bias. Standard axial CT scan for pulmonary embolism was carried out with collimation of $1.25 \mathrm{~mm}$, pitch of six and a reconstruction interval of $0.8 \mathrm{~mm}$. Paddle wheel reconstruction was carried out by using inbuilt software, setting $5 \mathrm{~mm}$ slab thickness and 5 degree rotation. Reference standard was overall interpretation of axial CT scans by both the radiologists.

Results: Sensitivity and specificity for axial images was between 90 to $100 \%$; sensitivity for paddle wheel technique ranged from 65 to $72 \%$ while specificity turned out to be 80 to $100 \%$. Final consensus agreement for standard axial imaging was higher than paddle wheel technique.

Conclusion: No significant difference between two imaging methods was noted in cases of central pulmonary embolism, however standard axial imaging was better than paddle wheel technique in detecting peripheral pulmonary embolism.
\end{abstract}

Keywords: Pulmonary embolism, computed tomography, pulmonary angiography, paddle wheel reformation, pulmonary arteries

\section{Introduction}

Pulmonary embolism is still a frequently encountered cause of patient's morbidity and mortality in all parts of the world [1]. Most of the deaths associated with this potentially fatal disease occur due to a missed diagnosis; the reason being its protean manifestations [2]. Ongoing researches are still trying to evolve a single most sensitive and specific imaging method to detect pulmonary embolism $[3,4]$.

Multi-detector helical CT technology has already achieved its role as standard modality of choice in patients with suspicion of pulmonary embolism [3-5]. Rapid scanning decreased motion artifacts with enhanced evaluation of vessels on one hand, while on other hand thinner collimation increased the work load by increasing the images for review [6].

Paddle wheel technique described by Simon et al., $[7,8]$ is a reconstruction method of displaying images, which uses planar slabs pivot on the central horizontal axis at bifurcation of pulmonary artery trunk. With this method, each image depicts complete track of branching structures from central hilum to the periphery.

Purpose of our study is twofold; to compare sensitivity and specificity of rotated paddle wheel technique to standard axial 
images for detection of both central and peripheral pulmonary embolism, and to assess all possible advantages of paddle wheel reformation so as to justify its inclusion in the standard protocol for pulmonary embolism in our department.

\section{Materials and methods}

This prospective study was carried out by including 50 adult subjects presenting with suspicion of pulmonary embolism from April 2013 to June 2014.

Of all these patients 40 were found to have acute pulmonary embolism on multi-detector helical CT using standard axial protocol. 31 patients were females and 19 were males. Age range was between 19 and 82 years (mean age 41.5 years).

All CT scans were performed using CT scanner (LightSpeed TM 7.X CT scanner, GE Medical systems, USA). Gantry position rotation period was $0.4 \mathrm{~s}$, with an $\mathrm{x}$-ray tube voltage of $140 \mathrm{Kv}$ and a current of 230-350 mA. Cranio-cuadal image acquisition of entire thorax was carried out with a collimation of $1.25 \mathrm{~mm}$, (pitch six), and a reconstruction increment of $0.8 \mathrm{~mm}$. Mean scan time was $2.30 \mathrm{sec}$. All patients underwent CTPA after administration of $100 \mathrm{ml}$ standard contrast media (Xenetix $350 \mathrm{mg} / \mathrm{ml}$, Guerbet, France) with an injection rate of 4.5-5 $\mathrm{ml} / \mathrm{s}$ via 16 gauge catheter inserted into ante cubital vein. Contrast bolus was followed by a $10 \mathrm{ml}$ saline flush using Enhanced Xtream injector (Nemoto Dual Shot Alpha CiA425 Class IV/GE) and delay was estimated using semiautomatic bolus tracking system (SmartPrep, GE Medical system, USA) with a threshold of $80 \mathrm{HU}$ for all examination. ROI for bolus tracking was measured in right atrium.

All images were sent to workstation (VEPRO, Germany). Reformations were performed by a senior technologist using a reconstruction program. Planning for maximum intensity projection (MIP) images in paddle wheel technique was done from the axial CT data. Axis of rotation was positioned at the bifurcation of pulmonary artery trunk, using left lateral medial sagittal scout image. Each planar slab had a thickness of $5 \mathrm{~mm}$. Angle between successive slabs was 5 degrees; the whole rotation coverage was 180 degrees. With this technique, total of 36 images covered the whole lung. The unique feature of the reformation was to exhibit branching vessels radiating from the hila without any discontinuity.

Image analysis was obtained on workstation (VEPRO, Germany), using standard mediastinal window setting (window width: $350 \mathrm{HU}$, window center: $50 \mathrm{HI}$ ).

Blind and random analysis of axial and reformatted images was done. One of the radiologist firstly reviewed axial images in 25 patients followed by reformatted images, however the other radiologist reviewed vice versa. To avoid any possible bias, an interval of two weeks was intentionally created between two readings.

Pulmonary embolism was defined as central or eccentric partial filling defect surrounded by contrast medium and occupying entire arterial vessel section [6]. Presence or absence of emboli in pulmonary artery or its branches was carried out by using nomenclature outlined by Remy-Jardin et al., [9] which is based on the standard descriptions by Boyden [10]. For images from each pulmonary CTA as a whole, readers used a scale of 5-point scale to score the embolus as follows: 5 , definitely present; 4 , probably present; 3 , indeterminate; 2 , probably absent; 1 , definitely absent. Another 3-point scale was formulated to score different anatomical levels of pulmonary arteries of each lobe: 1, central (main, right, and left); 2, lobar and 3, segmental.

Sub-segmental levels were not included in the analysis of this study. Reference standard was overall interpretation of axial CT scans by both radiologists.

\section{Statistical analysis}

Statistical analysis was done with SPSS, statistical package (SPSS, Version 17.0). Overall sensitivity and specificity of axial and reformatted CT images was calculated. Moreover sensitivity and specificity of axial and reformatted images for central and peripheral arteries was also calculated.

The linear, weighted $k$ statistic was applied for interobserver agreement between the two radiologists. The $k$ value range from -1 (no agreement) to 1 (almost perfect agreement). Agreement was rated poor $(k<0.00)$, slight $(0.00-0.20)$, fair $(0.21-0.40)$, moderate (0.1-0.60), substantial (0.61-0.80) or almost perfect $(>0.81)$ [11].

Chi-square test was used and $p$-values less than 0.05 were considered statistically significant.

\section{Results}

Out of 50 patients undergoing CT pulmonary angiography for suspected pulmonary embolism, 31 were females and 19 male subjects (Figure 1). Positive cases were confirmed in 13

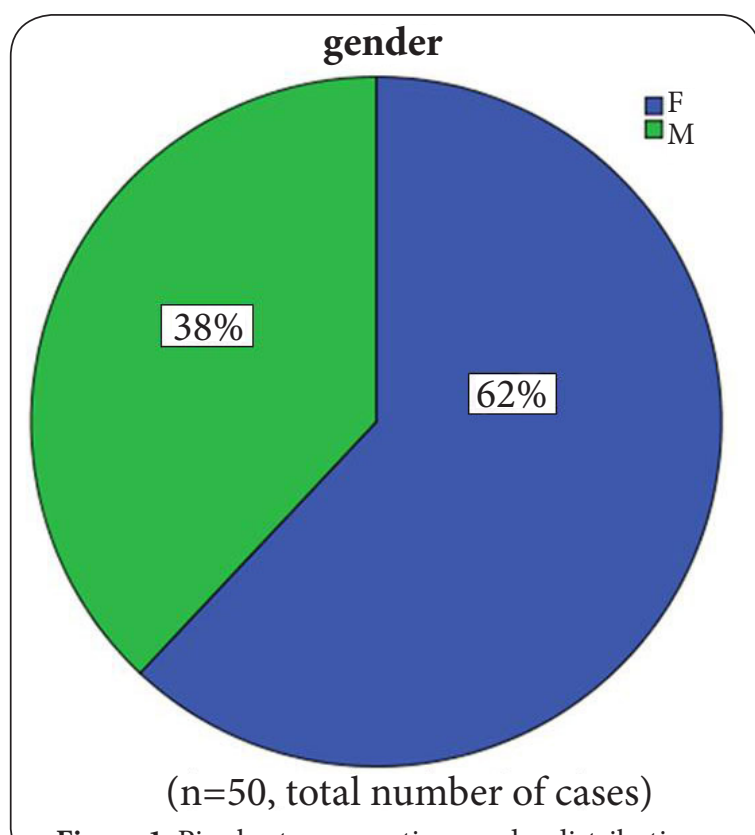

Figure 1. Pie chart representing gender distribution. 
of 19 male patients and 27 of 31 female patients. Youngest patient included in our study and found positive for pulmonary embolism was 18 years of age and oldest 82 years of age (Figure 2).

Considering the defined reference standard, total of 40 positive cases of pulmonary embolism were detected, including 18 central, 13 lobar and 9 peripheral emboli. All 40 cases were discovered by first radiologist, however second radiologist detected 36 cases of pulmonary embolism.

Association of positive D-dimers levels was also observed in patients presenting with suspicion of pulmonary embolism (Figure 3).

Forty ( 18 central/13 lobar/9 peripheral) clots were detected by first radiologist on standard axial CT images, however 26 (18 central/8 lobar) clots on paddle wheel reformatted images. Second radiologist read 35 (18 central/13 lobar/4 peripheral) emboli on standard axial images while 30 (18 central/9 lobar/ 3 peripheral) on paddle wheel technique (Table 1).
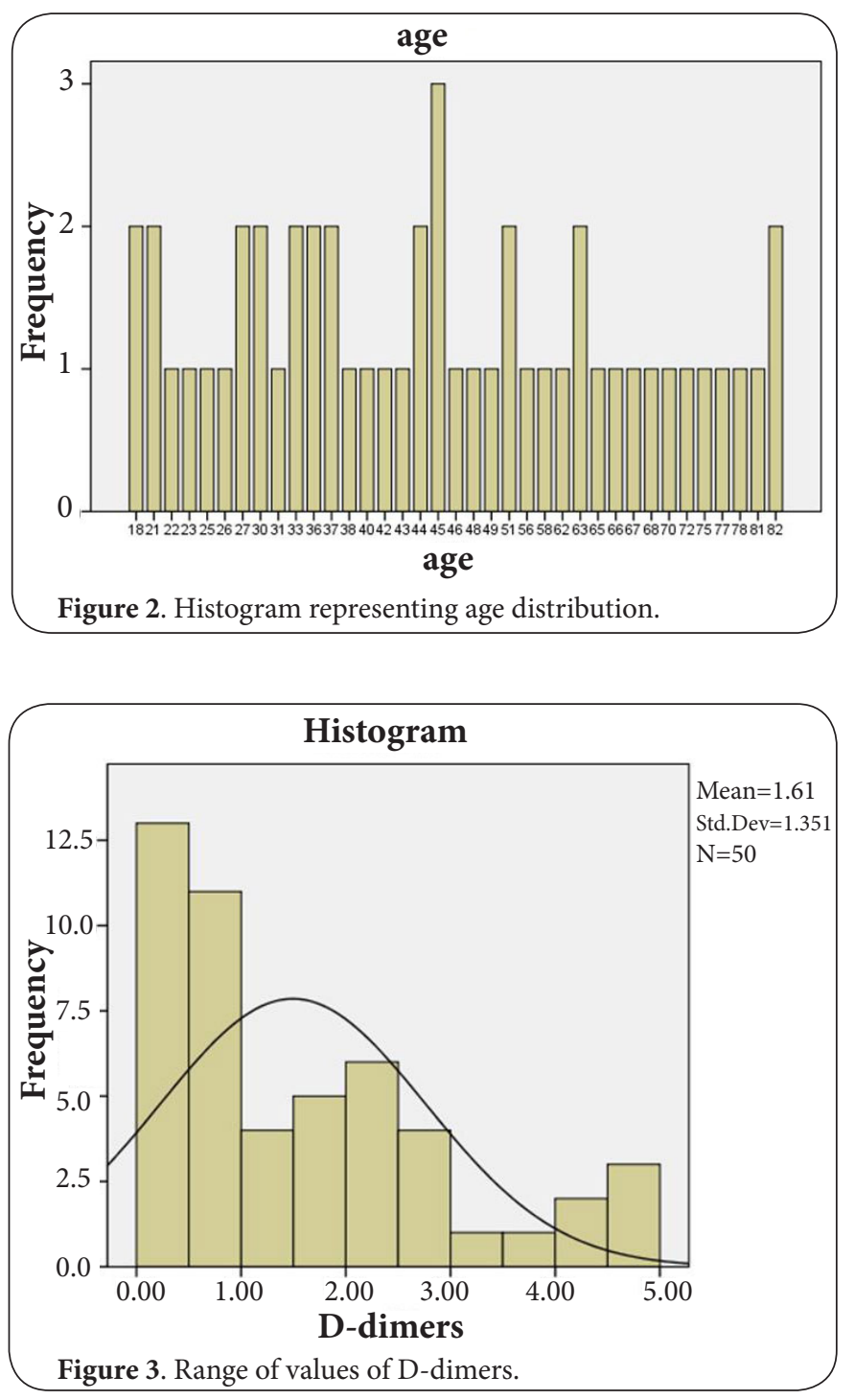

Final consensus agreement for standard axial imaging was higher than paddle wheel technique.

Sensitivity and specificity for detecting both central and peripheral emboli on axial images was between 90 to $100 \%$; however sensitivity for paddle wheel technique ranged from 65 to $72 \%$ while specificity turned out to be 80 to $100 \%$ (Table 2). $2 \%$ while specificity turned out to be 80 to $100 \%$ (Table 2).

Table 1. Number of emboli detected by axial and paddle wheel reformations.

\begin{tabular}{lllllll}
\hline & \multicolumn{3}{c}{ Axial images } & \multicolumn{3}{c}{ Paddle wheel reformations } \\
\hline & Central & Lobar & Segmental & Central & Lobar & Segmental \\
\hline $\begin{array}{l}\text { First } \\
\text { Radiologist }\end{array}$ & 18 & 13 & 9 & 18 & 13 & 4 \\
$\begin{array}{l}\text { Second } \\
\text { Radiologist }\end{array}$ & 18 & 8 & 0 & 18 & 9 & 3 \\
\hline
\end{tabular}

( $\mathrm{n}=50$, total number of cases)

Table 2. Sensitivity (\%) and specificity (\%) of axial and paddle wheel reformations.

\begin{tabular}{lllll}
\hline & \multicolumn{2}{c}{ Axial images } & \multicolumn{2}{c}{ Paddle wheel reformations } \\
\hline & Sensitivity & Specificity & Sensitivity & Specificity \\
\hline First Radiologist & 100 & 100 & 65 & 72 \\
Second Radiologist & 90 & 90 & 80 & 100 \\
\hline
\end{tabular}

( $\mathrm{n}=50$, total number of cases)

Interobserver agreement of overall detection of pulmonary embolism on standard axial images is 0.719 , and for paddle wheel technique is 0.715 which is substantial.

Sensitivity for paddle wheel technique was lowered as compared to standard axial scans, due to inability to detect peripheral emboli. However the specificity was substantially comparable to standard axial scans.

\section{Discussion}

Aim of our study was twofold; firstly to compare the sensitivity and specificity of paddle wheel technique in evaluation of pulmonary embolism as compared to standard axial CT imaging; secondly to assess all possible advantages of paddle wheel reformation so as to justify its inclusion in the standard protocol for pulmonary embolism in our department.

In our experience, paddle wheel technique was easily implemented on our CT equipment (LightSpeed ${ }^{\mathrm{TM}}$ 7.X CT scanner, GE Medical systems, USA). Our multi detector CT scanner easily carried out reformation using the technique with high quality images produced in few minutes. Protocol was simple and the technician grasped it rapidly with already in built software in our scanner. As a whole the experience to evaluate a new method helped us to assess other facts and advantages about the technique.

These reformations provided a unique way to trace the branching arteries from the hilum towards the periphery 
Kamel et al. Medical Imaging and Radiology 2014,

http://www.hoajonline.com/journals/pdf/2054-1945-2-4.pdf

of the lung without any discontinuity (Figures 4A and 4B). Moreover the continuous display of the vessel enabled the observer to assess the embolus in detail.

When we compare the overall detection of central pulmonary embolism by standard axial CT images with paddle wheel reformation method (Figures 5,6 and 7), there was no significant difference between the two. However, paddle wheel technique did show less percentage of peripheral emboli as compared to standard axial images routinely carried
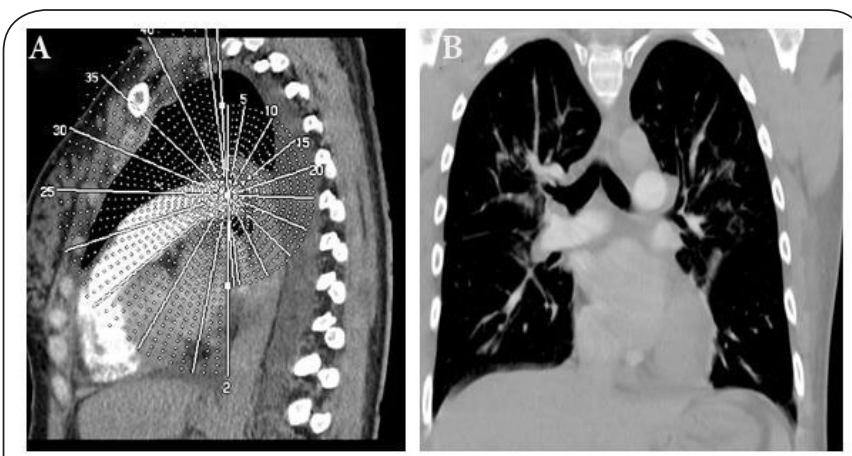

Figure 4. (A) Lateral medial sagittal scout image with the axis of rotation and rotating slabs used for paddlewheel planning with central axis at the main pulmonary artery $(5 \mathrm{~mm}$ slab thickness, $5^{\circ}$ rotation). (B) The reformatted image.
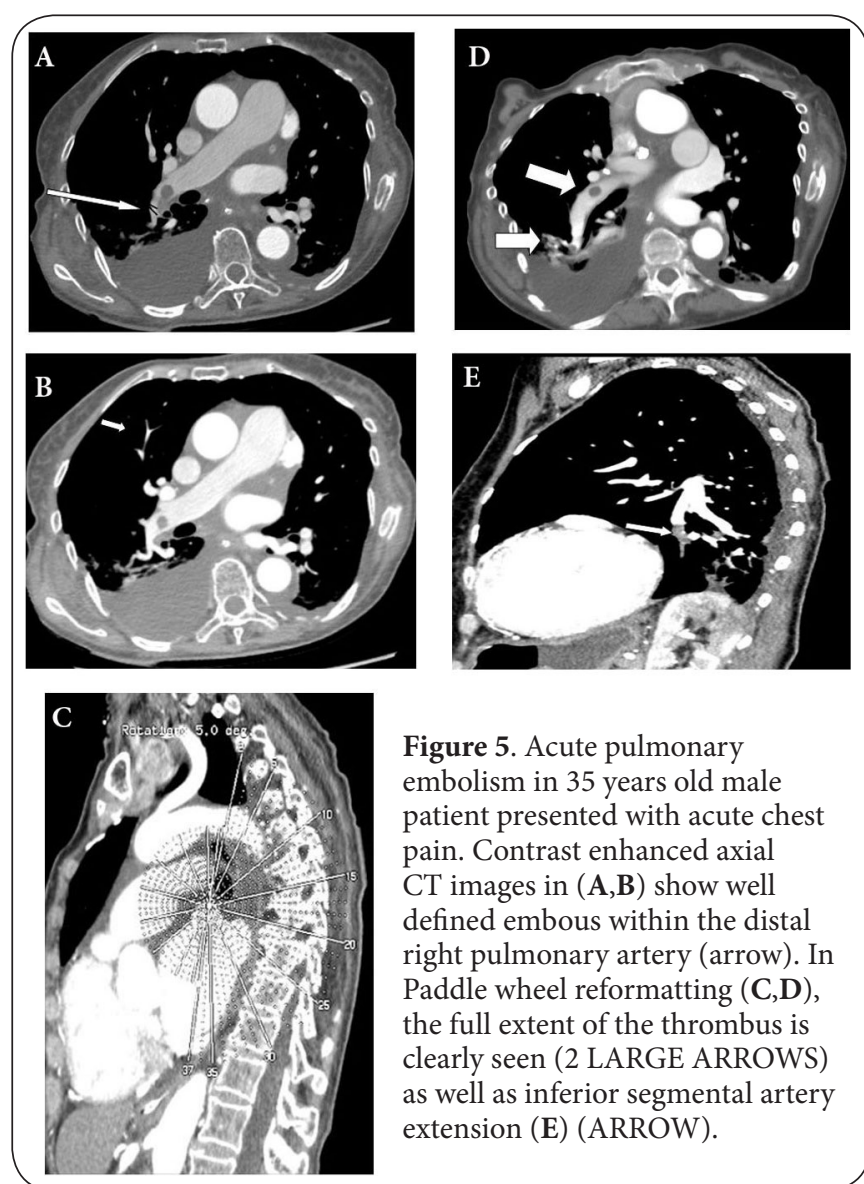

Figure 5. Acute pulmonary embolism in 35 years old male patient presented with acute chest pain. Contrast enhanced axial CT images in $(\mathbf{A}, \mathbf{B})$ show well defined embous within the distal right pulmonary artery (arrow). In Paddle wheel reformatting $(\mathbf{C , D})$, the full extent of the thrombus is clearly seen (2 LARGE ARROWS) as well as inferior segmental artery extension (E) (ARROW).
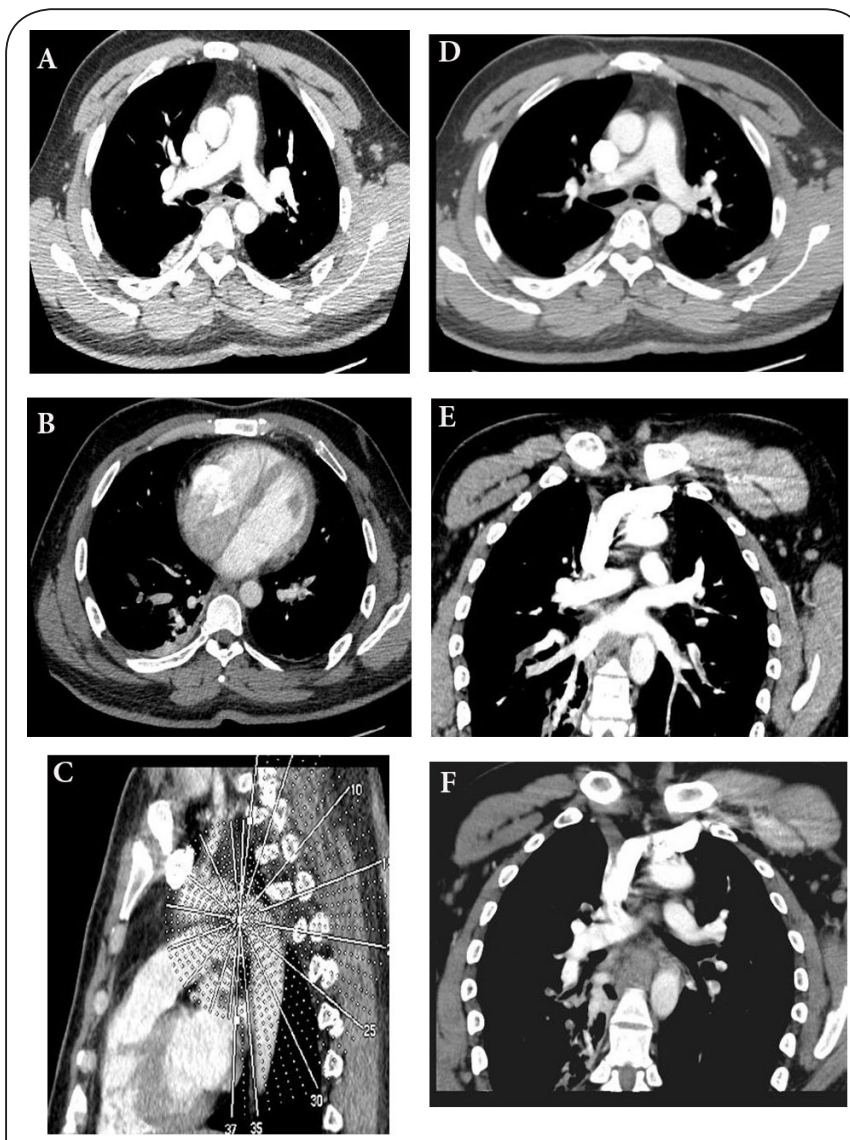

Figure 6. 45 years old female patient presented with acute chest pain. Contrast enhanced axial CT images (A) show normally enhancing main \& proximal right \& left pulmonary artery with bilateral non enhancing sub-segmental emboli (B). In paddle wheel reformatting $(\mathbf{C}, \mathbf{D})$, the emboli are visualized within the distal right main artery \& inferior segmental arteries on both sides. In (E,F), the non enhancing emboli within the inferior segmental \& sub-segmental branches are seen in continuity.

out in our department. The result of our study has shown significantly high sensitivity and specificities for detecting emboli. Moreover the inter-observer agreement for central emboli is high.

Our study has certain limitations. To start with the most important, we did not include conventional pulmonary angiography as a gold standard. In our hospital, the procedure is rarely performed and we could not use it as a reference. We kept the axial CT images as standard and compared a new reformation technique against a routine protocol of our department. Overall we tried to assess the percentage of positive cases detected with each method. Secondly, our study was carried out on small sample volume $(n=50)$ which lowers the statistical significance of the study. However including discretely obvious cases of pulmonary embolism did helped in compensating the results statistically.

Although few aspects of paddle wheel reformation were 

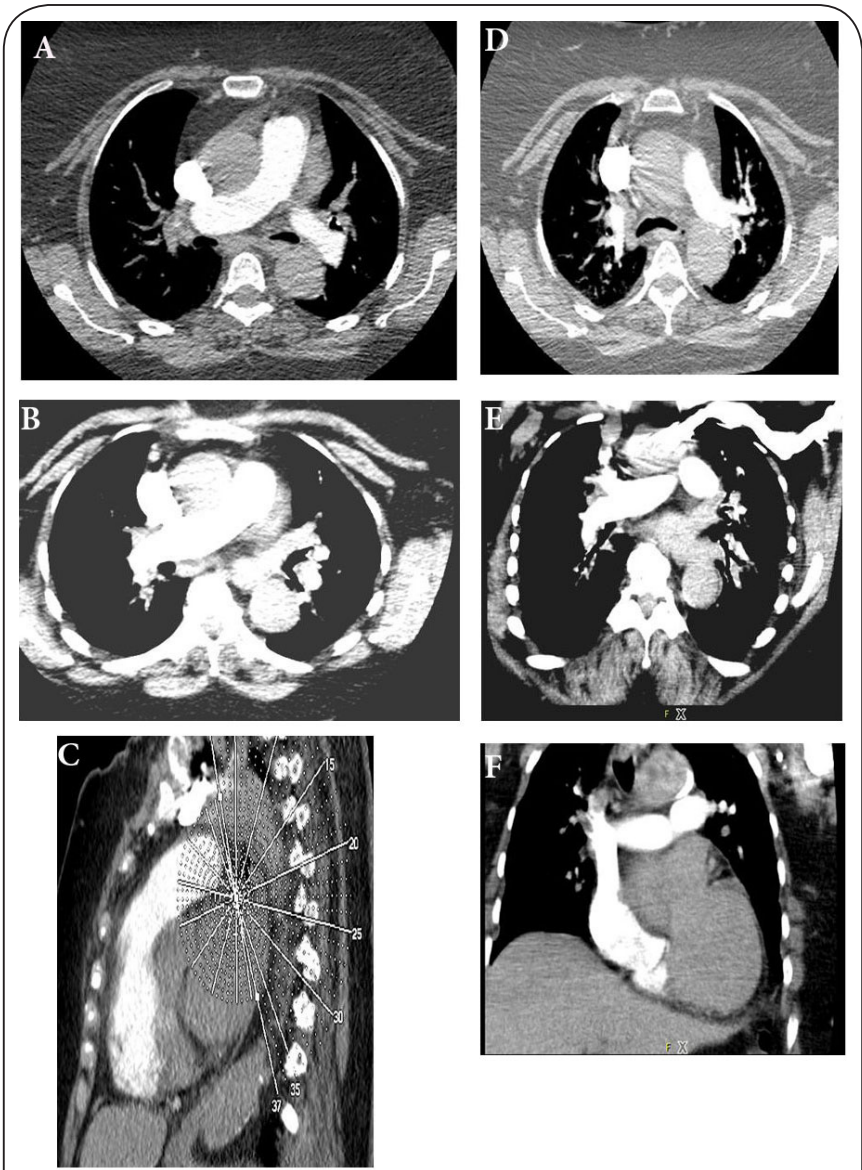

Figure 7.51 years old female patient presented with acute chest pain. Contrast enhanced Axial CT images $(\mathbf{A}, \mathbf{B})$ show non enhancing segmental emboli on both sides. In Paddle Wheel Reformatting (C,D,E and F) (5 mm slap thickness, 5 degree rotation) separate visualization of left \& right pulmonary arteries seen in continuity from the hilum to the periphery with the emboli seen within the segmental \& subsegmental branches.

assessed, but the results of our study showed its complementary role to standard axial CT.

\section{Conclusions}

Both paddle wheel technique and standard axial CT displayed significantly high sensitivity and specificity in detecting central pulmonary embolism, however paddle wheel technique showed less percentage of peripheral emboli as compared to standard axial images. Moreover, unique continuous display of pulmonary arteries and detailed assessment of embolus proved to be additional diagnostic advantage. At this stage may be it will not be possible to include the paddle wheel technique in routine protocol of our department, but based on this pioneer study, we would be able to explore unique strengths of this reformatting method in future studies.

\section{Competing interests}

The authors declare that they have no competing interests.
Authors' contributions

\begin{tabular}{|l|c|c|c|}
\hline Authors' contributions & HSD & HAK & SRQ \\
\hline Research concept and design & $\checkmark$ & $\checkmark$ & $\checkmark$ \\
\hline Collection and/or assembly of data & $\checkmark$ & $\checkmark$ & $\checkmark$ \\
\hline Data analysis and interpretation & $\checkmark$ & $\checkmark$ & $\checkmark$ \\
\hline Writing the article & $\checkmark$ & $\checkmark$ & $\checkmark$ \\
\hline Critical revision of the article & $\checkmark$ & $\checkmark$ & $\checkmark$ \\
\hline Final approval of article & $\checkmark$ & $\checkmark$ & -- \\
\hline Statistical analysis & -- & -- & $\checkmark$ \\
\hline
\end{tabular}

Acknowledgement

The authors would like to thank their colleagues for the support and patients for their trust.

\section{Publication history}

Senior Editor: Domenico Rubello, Santa Maria della Misericordia Hospital, Italy.

Received: 09-Jun-2014 Final Revised: 28-Aug-2014

Accepted: 07-Sep-2014 Published: 15-Sep-2014

\section{References}

1. Ginsberg JS, Wells PS, Brill-Edwards P, Donovan D, Panju A, van Beek EJ and Patel $A$. Application of a novel and rapid whole blood assay for D-dimer in patients with clinically suspected pulmonary embolism. Thromb Haemost. 1995; 73:35-8. | Article I PubMed

2. Wells PS, Ginsberg JS, Anderson DR, Kearon C, Gent M, Turpie AG, Bormanis J, Weitz J, Chamberlain M, Bowie D, Barnes D and Hirsh J. Use of a clinical model for safe management of patients with suspected pulmonary embolism. Ann Intern Med. 1998; 129:997-1005. | Article | PubMed

3. Rubin GD, Shiau MC, Leung AN, Kee ST, Logan LJ and Sofilos MC. Aorta and iliac arteries: single versus multiple detector-row helical CT angiography. Radiology. 2000; 215:670-6. | Article | PubMed

4. Raptopoulos V and Boiselle PM. Multi-detector row spiral CT pulmonary angiography: comparison with single-detector row spiral CT. Radiology. 2001; 221:606-13. | Article | PubMed

5. Goodman LR, Lipchik RJ, Kuzo RS, Liu Y, McAuliffe TL and O'Brien DJ. Subsequent pulmonary embolism: risk after a negative helical CT pulmonary angiogram--prospective comparison with scintigraphy. Radiology. 2000; 215:535-42. | Article | PubMed

6. Winer-Muram HT, Rydberg J, Johnson MS, Tarver RD, Williams MD, Shah H, Namyslowski J, Conces D, Jennings SG, Ying J, Trerotola SO and Kopecky KK. Suspected acute pulmonary embolism: evaluation with multi-detector row CT versus digital subtraction pulmonary arteriography. Radiology. 2004; 233:806-15. | Article | PubMed

7. Simon M, Chiang EE and Boiselle PM. Paddle-wheel multislice helical CT display of pulmonary vessels and other lung structures. Radiol Clin North Am. 2003; 41:617-26. | Article | PubMed

8. Simon M, Boiselle PM, Choi JR, Rosen MP, Reynolds K and Raptopoulos V. Paddle-wheel CT display of pulmonary arteries and other lung structures: a new imaging approach. AJR Am J Roentgenol. 2001; 177:195-8. | Article | PubMed

9. Remy-Jardin M, Remy J, Artaud D, Deschildre F and Duhamel A. Peripheral pulmonary arteries: optimization of the spiral CT acquisition protocol. Radiology. 1997; 204:157-63. | Article | PubMed

10. Boyden EA. Segmental Anatomy of the Lungs. New York, NY: McGrawHill: 1955. 
Kamel et al. Medical Imaging and Radiology 2014,

http://www.hoajonline.com/journals/pdf/2054-1945-2-4.pdf

11. Cicchetti DV and Feinstein AR. High agreement but low kappa: II.

Resolving the paradoxes. J Clin Epidemiol. 1990; 43:551-8. | Article |

PubMed

\section{Citation:}

Kamel HA, Darwish HS and Qamar SR. Comparison of rotated paddle wheel reformation with standard axial CT scan in pulmonary embolism; a hospital based study. Med Imaging Radiol. 2014; 2:4.

http://dx.doi.org/10.7243/2054-1945-2-4 